ARTICLE

Received 2 Dec 2013 | Accepted 14 Dec 2014 | Published 4 Feb 2015

DOI: $10.1038 /$ ncomms7095 OPEN

\title{
The jasmonate-responsive GTR1 transporter is required for gibberellin-mediated stamen development in Arabidopsis
}

Hikaru Saito1, Takaya Oikawa², Shin Hamamoto ${ }^{3}$, Yasuhiro Ishimaru², Miyu Kanamori-Sato1, Yuko Sasaki-Sekimoto ${ }^{4}$, Tomoya Utsumi ${ }^{1}$, Jing Chen ${ }^{1}$, Yuri Kanno ${ }^{5}$, Shinji Masuda ${ }^{4,6}$, Yuji Kamiya ${ }^{5}$, Mitsunori Seo ${ }^{5}$, Nobuyuki Uozumi ${ }^{3}$, Minoru Ueda ${ }^{2} \&$ Hiroyuki Ohta ${ }^{1,4}$

Plant hormones are transported across cell membranes during various physiological events. Recent identification of abscisic acid and strigolactone transporters suggests that transport of various plant hormones across membranes does not occur by simple diffusion but requires transporter proteins that are strictly regulated during development. Here, we report that a major glucosinolate transporter, GTR1/NPF2.10, is multifunctional and may be involved in hormone transport in Arabidopsis thaliana. When heterologously expressed in oocytes, GTR1 transports jasmonoyl-isoleucine and gibberellin in addition to glucosinolates. gtr1 mutants are severely impaired in filament elongation and anther dehiscence resulting in reduced fertility, but these phenotypes can be rescued by gibberellin treatment. These results suggest that GTR1 may be a multifunctional transporter for the structurally distinct compounds glucosinolates, jasmonoyl-isoleucine and gibberellin, and may positively regulate stamen development by mediating gibberellin supply.

\footnotetext{
${ }^{1}$ Graduate School of Bioscience and Biotechnology, Tokyo Institute of Technology, 4259-B65 Nagatsuta-cho Midori-ku, Yokohama 226-8501, Japan.

${ }^{2}$ Graduate School of Science, Tohoku University, 6-3, Aramaki-Aza-Aoba, Aoba-ku, Sendai 980-0845, Japan. ${ }^{3}$ Graduate School of Engineering, Tohoku University, 6-6-07, Aobayama, Aoba-ku, Sendai 980-8579, Japan. ${ }^{4}$ Earth-Life Science Institute, Tokyo Institute of Technology, 2-12-1-IE-1 Ookayama, Meguro-ku, Tokyo 152-8551, Japan. ${ }^{5}$ RIKEN Center for Sustainable Resource Science, 1-7-22 Suehiro-cho Tsurumi-ku, Yokohama 230-0045, Japan. ${ }^{6}$ Center for Biological Resources and Informatics, Tokyo Institute of Technology, 4259-B65 Nagatsuta-cho Midori-ku, Yokohama 226-8501, Japan. Correspondence and requests for materials should be addressed to H.O. (email: hohta@bio.titech.ac.jp).
} 
$\mathrm{P}$ lant hormones are signalling molecules that induce a wide spectrum of physiological responses at extremely low concentrations. Both import and export of plant hormones across the plasma membrane are important steps preceding intraand extracellular responses. Hormone transport across the plasma membrane does not take place by simple diffusion but generally requires transporter proteins. In plants, hormone transport is important for controlling various physiological responses. Recent studies have identified several hormone-transporting proteins. Auxin transporters have been identified by mutant analysis based on abnormal phenotypes in their organ development or responses to exogenous auxins or environmental stimuli ${ }^{1}$. Another study identified a different type of auxin transporter, NRT1.1/NPF6.3, which belongs to the NITRATE TRANSPORTER 1/PEPTIDE TRANSPORTER FAMILY (NPF) ${ }^{2,3}$. NRT1.1/NPF6.3 facilitates the uptake of auxin, and nitrate inhibits this uptake ${ }^{3}$. Two ATPbinding cassette $(\mathrm{ABC})$-type transporters were identified as abscisic acid (ABA) transporters; AtABCG25 mediates ABA export from cells, and AtABCG40 mediates ABA uptake ${ }^{4,5}$.

A modified yeast two-hybrid system was used to identify another type of ABA transporter, AIT1/NPF4.6, which also belongs to the NPF family ${ }^{4}$ AIT1/NPF4.6 mutants are less sensitive to exogenously applied ABA during seed germination and/or post-germination growth ${ }^{6}$. A DTX/multidrug and toxic compound extrusion family member; AtDTX50 functions as an ABA efflux transporter ${ }^{7}$. An ATP-binding cassette transporter in Arabidopsis, AtABCG14, is essential for the translocation of the root-synthesized cytokinins ${ }^{8,9}$. The ABC transporter PDR1 in Petunia hybrida was identified as a key regulator in the initiation of arbuscular mycorrhizae and axillary branches by functioning as a cellular strigolactone exporter ${ }^{10}$.

Movement of gibberellin (GA) and jasmonates (JAs) may also be important in various physiological events including seed germination, flower induction and development ${ }^{11-18}$. However, transporters for these hormones have not been identified except for the in vitro GA transport activity of AIT3/NPF4.1 (ref. 6). In plants, JAs are $\alpha$-linolenic acid-derived hormones that regulate a wide spectrum of plant processes such as pollen and stamen maturation, senescence and defence responses against biotic and abiotic stresses ${ }^{14-16}$. In particular, jasmonoyl-isoleucine (JA-Ile) is a bioactive form of JA that is involved in various physiological responses ${ }^{14}$. JA-deficient Arabidopsis mutants are male sterile because of defects in filament elongation, completion of anthesis and anther dehiscence ${ }^{17,18}$. Stamen development is also regulated by GA, which is essential for not only floral development but also seed germination, shoot and root growth, and fruit and seed development ${ }^{11-13}$. The GA-deficient mutant ga1-3 is a male sterile dwarf, and the phenotype can be converted to the wild type (WT) by repeated application of $\mathrm{GA}^{19-21}$. Anatomical analysis of this mutant revealed that the male sterile phenotype is because of the arrest of stamen filament cell elongation and failure to complete anthesis ${ }^{22}$. These findings indicate the importance of JA and GA in flower and seed development.

Recently, some details of JA-GA crosstalk have emerged, and the interplay between these two hormones regulates growth and defence ${ }^{23-25}$. DELLA proteins, which are negative regulators of GA signalling, directly interact with JAZ (jasmonate ZIMdomain) repressors of the JA-responsive transcription factor MYC2, suggesting that DELLAs compete with MYC2 for binding to JAZ and modulate JA signalling ${ }^{23}$. Another study showed that sesquiterpene synthase genes are synergistically induced by JA and GA in inflorescences. DELLAs directly interact with MYC2, which promotes the transcription of genes encoding sesquiterpene biosynthetic genes, suggesting that JA and GA promote sesquiterpene biosynthesis via degradation of JAZs and DELLAs, respectively, which are negative regulators of MYC2 (ref. 24). Both JA and GA are known to be important for various developmental processes and accumulating evidence indicates the existence of JA-GA crosstalk in their signalling. However, functional interplay of JA and GA in a specific developmental stage is still largely unknown.

Gene network analysis is an effective way to find sets of genes, which are functionally correlated in some signalling processes ${ }^{26}$. Here we identified the JA-responsive NPF family protein, GTR1/ NPF2.10, using co-expression analysis of JA-responsive genes. The GTR1 protein showed GA and JA-Ile transport activities, and the gtr1 mutant showed decreased fertility owing to impaired stamen development. Supplementation with bioactive GAs at the flowering stage, however, fully restored the phenotype to that of the wild type (WT). These results suggest that GTR1/NPF2.10 may be involved in stamen development via active GA supply.

\section{Results}

Identification of GTR1. To identify a novel regulator(s) of JA signalling, we searched co-expressed genes of nine jasmonate biosynthetic genes (DAD1, LOX3, LOX4, AOS, AOC3, AOC4, OPR3, OPCL1 and JAR1) using ATTED-II CoExSearch ver. c4.1. (http://atted.jp) ${ }^{27}$. We selected genes, which co-expressed with JA biosynthetic genes in the top 20 mutual rank of the Pearson's correlation coefficient ${ }^{28}$ (Supplementary table 1). We found several genes, as well as $J A Z$ gene family and transcription factors, simultaneously expressed with JA biosynthetic genes. A unique NPF family gene, AT3G47960/GTR1/NPF2.10, which encodes a glucosinolate transporter ${ }^{29}$, was tightly co-expressed with JA biosynthesis genes such as $L O X 3, A O S, O P R 3$ and JAR1. A recent study on NRT1.1/NPF6.3, which belongs to the same transporter family as GTR1/NPF2.10, showed that the protein is an auxin transporter ${ }^{3}$. Another study on NPF family showed that AIT1/ NPF4.6 is an ABA transporter ${ }^{6}$. This study also showed that another NPF protein, AIT3/NPF4.1, has ABA and GA transport activities, suggesting that GTR1/NPF2.10 may transport JAs or other hormones.

GTR1 is JA-responsive and regulates JA-related genes. To analyse the response of GTR1 to JAs in detail, we treated liquidcultured 10-day-old Arabidopsis seedlings with $20 \mu \mathrm{M}$ methyl jasmonate (MeJA) and then analysed GTR1 transcript levels with quantitative PCR with reverse transcription (RT-PCR). A previous study indicated that $M Y C 2$ and JAZ family genes are rapidly induced by $\mathrm{MeJA} \mathrm{A}^{30-33}$. GTR 1 was increased in response to MeJA in $30 \mathrm{~min}$, and high expression was maintained for $24 \mathrm{~h}$ after treatment (Fig. 1a). We also analysed GTR1 expression upon cycloheximide (CHX) treatment to test whether the expression requires protein synthesis. GTR 1 was upregulated more so and at a later time in the presence of $\mathrm{CHX}+\mathrm{MeJA}$ compared with single treatment with MeJA, and GTR1 expression also increased upon treatment with CHX alone (Fig. 1a). These data suggest that the transient GTR1 expression is controlled by a primary responsive repressor whose synthesis is blocked by $\mathrm{CHX}$, resulting in prolonged GTR1 induction. Next, to elucidate functions of GTR1 in JA responses, we obtained T-DNA insertion lines and confirmed the insertion sites with PCR and sequence analyses (Supplementary Fig. 1a). We did not observe expression of the GTR1 coding region in the gtr1 mutant (Supplementary Fig. 1b). Thus, we considered gtr 1 to be a knockout mutant.

To clarify GTR1 function in JA signalling, we analysed expression of JA-responsive genes. In gtr 1 mutants, PDF1.2, a marker gene for jasmonate responses, was highly expressed in the absence of MeJA and did not respond to MeJA treatment for $24 \mathrm{~h}$ and kept higher expression level at $24 \mathrm{~h}$ after MeJA treatment, whereas PDF1.2 was induced by MeJA in WT plants (Fig. 1b). 

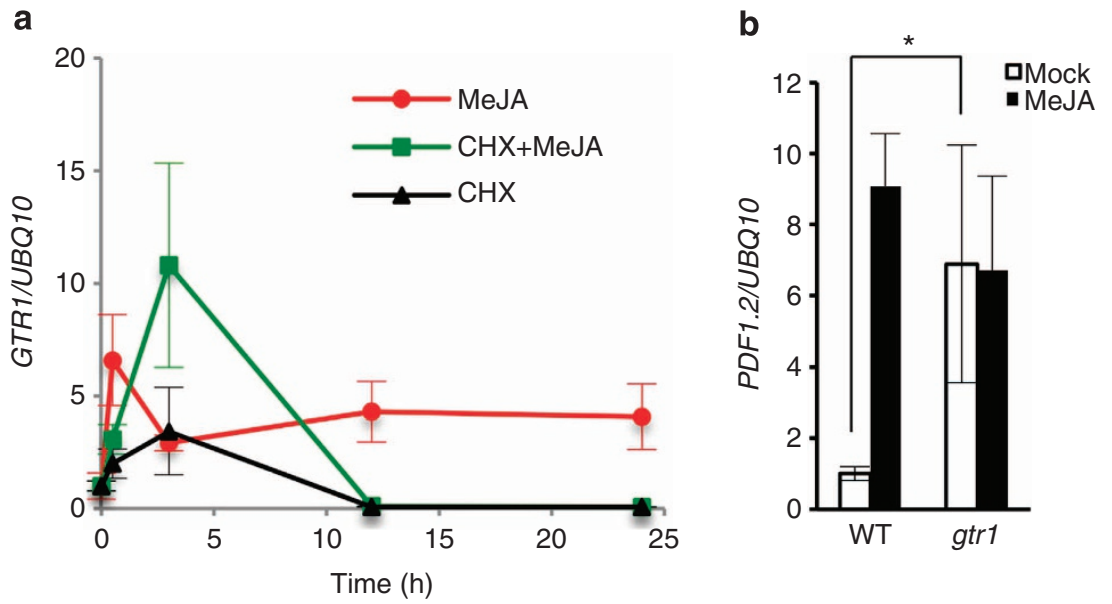

Figure 1 | GTR1 is induced by JA and regulates a JA-related gene. (a) Effect of cycloheximide (CHX) on MeJA induction. Ten-day-old liquid-cultured Arabidopsis Columbia WT was treated with or without $100 \mu \mathrm{M} \mathrm{CHX}$ for $1 \mathrm{~h}$ before treatment with or without $20 \mu \mathrm{M}$ MeJA for the number of hours indicated, followed by quantitative RT-PCR of GTR1 transcript levels. UBQ10 was used as a reference gene. Relative gene expression was calculated by normalizing to the value of mock-treated plants $(0.02 \%$ ethanol) for $0 \mathrm{~h}$. Values are the mean $\pm \mathrm{s}$.d. of three biological replicates. (b) Expression of PDF1.2 in gtr1 seedlings. Ten-day-old liquid-cultured Arabidopsis Columbia WT or gtr1 seedlings were treated with $0.02 \%$ ethanol (white bars) or $20 \mu \mathrm{M}$ MeJA (black bars) for $24 \mathrm{~h}$, followed by quantitative RT-PCR. UBQ10 was used as a reference gene. Relative gene expression was calculated by normalizing to the value of WT plants treated with $0.02 \%$ ethanol. Values are the mean \pm s.d. of three biological replicates. ${ }^{\star} P<0.05 ;$ Tukey-Kramer comparison test.

MYC2 expression in the mock-treated gtr1 mutant was about half that of WT, but expression of other JA-responsive genes including VSP1, ERF1 and ORA59 did not differ between WT and the gtr1 mutant (Supplementary Fig. 2). We also analysed the expression of JA biosynthesis genes in gtr1, and those levels were almost similar to WT except for LOX3 and JMT (Supplementary Fig. 3). These data suggest that a portion of JA signalling is disrupted in the gtr1 mutant.

The gtr1 mutation affects sensitivity to JA. We created 35S:GTR1/gtr1 lines. Complementation of GTR1 expression was confirmed with quantitative RT-PCR (Supplementary Fig. 1c). To investigate the sensitivity to MeJA, we grew plants on Murashige and Skoog (MS) medium with or without $50 \mu \mathrm{M}$ MeJA for 4 weeks. Remarkably, gtr1 plants turned brown after MeJA treatment, whereas WT plants remained green. 35S:GTR1/gtr1 plants complemented this aspect of the gtr1 phenotype (Fig. 2a). Plants grown on normal MS medium exhibited no visible differences among WT, gtr1 and 35S:GTR1/gtr1 plants (Fig. 2a). Next, to clarify whether this de-greening was due to accelerated leaf senescence, we analysed the expression of senescence marker genes $^{34}$ in gtr1. SAG20 was more highly expressed in the MeJAtreated gtr1 mutant compared with WT, but this was not the case for the mock-treated plants; for SAG12, another senescence marker, expression did not differ in the MeJA-treated plants but was significantly higher in mock-treated gtr1 plants compared with WT (Fig. 2b). The expression levels of SEN4 and SAG18 in gtr1 were comparable to that of MeJA- or mock-treated WT plants, respectively. The upregulated expression of SAG20 after MeJA treatment suggests that the de-greening phenotype in gtr 1 after MeJA treatment is due to the promotion of JA-induced premature leaf senescence.

GTR1 positively regulates stamen development. gtr1 plants have small siliques and reduced fertility, but the relative infertility could be partially reversed upon expression of 35S:GTR1/gtr1 (Fig. 3a). gtr1 had shorter stamen filaments than the WT and failed in anther dehiscence (Fig. 3b). We also pollinated gtr1 stigmas with WT pollen (WT* gtr1, Fig. 3c), which yielded mature siliques that were slightly longer than those of WT $(12.50 \pm 1.56$ for $\mathrm{WT}^{\star} g t r 1$ and $10.50 \pm 0.85$ for mock-treated WT, error bars are s.d., $n=10$; Table 1 ), and containing a similar number of seeds compared with WT $\left(34 \pm 11.2\right.$ for $\mathrm{WT}^{*}$ gtr 1 and $40.3 \pm 5.9$ for mock-treated WT, error bars are s.d., $n=10$; Table 2). To test the viability of mature pollen grains, we measured their germination rate; gtr1 and WT grains had similar germination rates (Supplementary Fig. 4). These data suggest that pollen rarely reached the stigma of gtr 1 plants and that the reduced fertility was caused by a defect in stamen development. Because JA coordinates stamen filament elongation, anther dehiscence and pollen viability at specific stages of flower development ${ }^{15,18}$, GTR1 may also regulate stamen development during JA signalling. Indeed, ProGTR1: $\beta$-glucuronidase (GUS) plants exhibited GUS staining in anthers, filaments and the base of filaments, demonstrating the expression of GTR1 in these floral organs (Fig. 4a).

We observed GUS staining in pollen of stage 12 flowers, but the staining was weak in vascular bundles (Fig. 4b,e). We observed marked GUS staining in vascular bundles of stage 13-14 filaments (Fig. 4c,d,f), at which time filament elongation occurs $^{35,36}$.

GTR1 imports JA-Ile and GA into Xenopus oocytes. Previous studies demonstrated that both GTR1/NPF2.10 and its homologue GTR2/NPF2.11 import glucosinolates, which are major Arabidopsis defence compounds, into Xenopus oocytes ${ }^{29}$. In addition, the gtrlgtr2 double mutant does not accumulate glucosinolates in seeds and accumulates excess glucosinolates in source tissues such as leaves and silique walls ${ }^{29}$. However, various gtr1 phenotypes noted in Figs 1-4 did not seem to be caused by a defect in glucosinolate transport. A recent study on NRT1.1/ NPF6.3, which belongs to the same transporter family (NRT1/ PTR Family, NPF) as GTR1/NPF2.10, showed that this protein is an auxin transporter ${ }^{3}$. Another study on AIT1/NPF4.6, which also belongs to the NPF family, showed that this protein is an ABA transporter ${ }^{6}$. That study also showed that another NPF protein, AIT3/NPF4.1, has ABA and GA transport activities ${ }^{6}$, raising the possibility that GTR1/NPF2.10 transports certain phytohormones across the plasma membrane where the protein is 
a

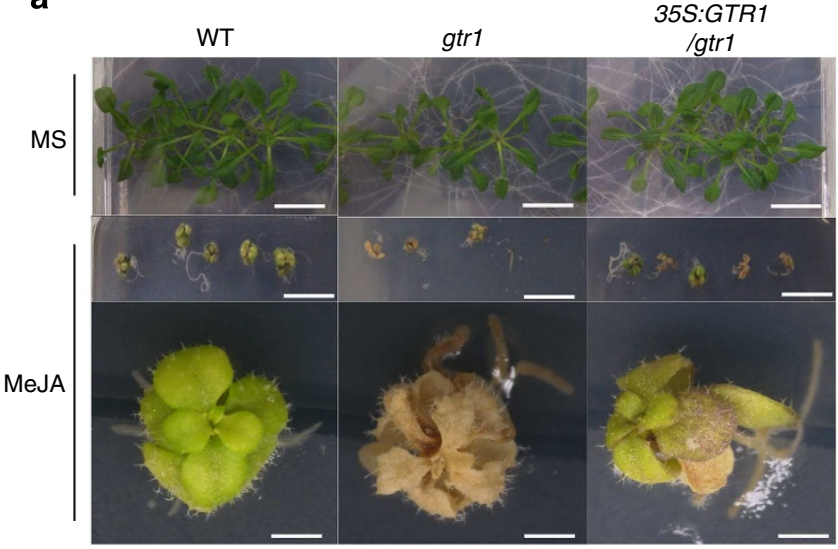

b
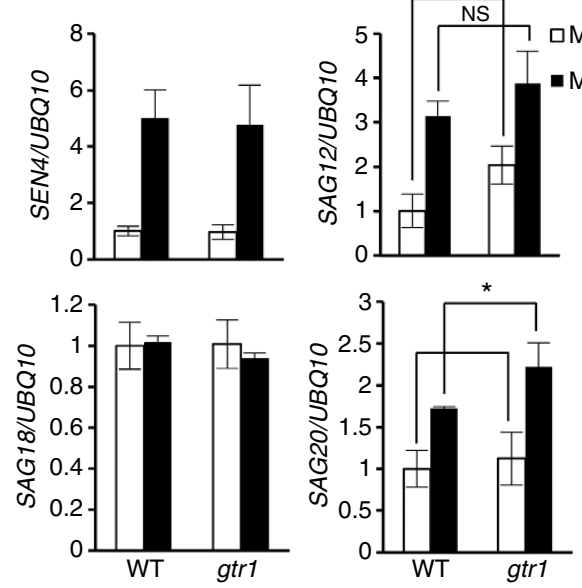

Figure 2 | The gtr1 mutant is hypersensitive to JA. (a) Phenotype of 30-day-old seedlings grown on Murashige and Skoog (MS) medium without (top panels) or with (middle panels) MeJA $(50 \mu \mathrm{M})$. The bottom panels show enlarged images of the seedlings in the second row. Scale bar, $10 \mathrm{~mm}$ (top and middle panels), $1 \mathrm{~mm}$ (bottom panels). (b) Expression of senescence-associated genes in gtr1 seedlings. Ten-day-old liquid-cultured Arabidopsis Columbia WT or gtr 1 seedlings were treated with $0.02 \%$ ethanol (white bars) or $20 \mu \mathrm{M}$ MeJA (black bars) for $72 \mathrm{~h}$, followed by quantitative RT-PCR. UBQ10 was used as a reference gene. Values are the mean \pm s.d. of three biological replicates. ${ }^{\star} P<0.05, N S$, not significant $(P>0.05)$; Student's $t$-test.

located (Supplementary Fig. 5). In addition, gtr1 showed decreased fertility resulting from a defect in stamen development (Fig. 3b), so we hypothesized that GTR1 could transport JA or GA, which regulate stamen development ${ }^{11,15,18}$. We therefore assessed the ability of GTR1 to import several plant hormones in Xenopus oocytes (Fig. 5). When JA, GA 3 , ABA, JAIle or tuberonic acid (TA) ${ }^{15}$ was added to the outside of GTR1expressing oocytes, $\mathrm{GA}_{3}$ and JA-Ile accumulated inside cells, whereas the other phytohormones did not (Fig. 5a). GA 3 also accumulated in oocytes expressing AIT3/NPF4.1 (Supplementary Fig. 6a), consistent with the report by Kanno et al. ${ }^{6}$ Plotting substrate uptake as a function of $\mathrm{GA}_{3}$ concentration yielded a saturation curve, which was best fitted by a Michaelis-Menten equation; this yielded an apparent affinity constant of $301.25 \pm 225.46 \mu \mathrm{M}$ (error bars are s.d., $n=5$ ) for $\mathrm{GA}_{3}$ transport by GTR1 (Supplementary Fig. 7). We also analysed GTR1 transport activity in the presence of the phytohormones and 4-methylthiobutyl glucosinolate (4MTB; a glucosinolate with high affinity for GTR1) ${ }^{29}$ and found specific accumulation of a

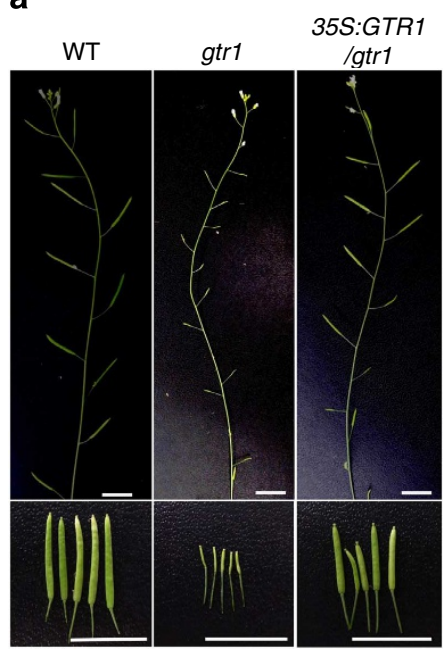

b

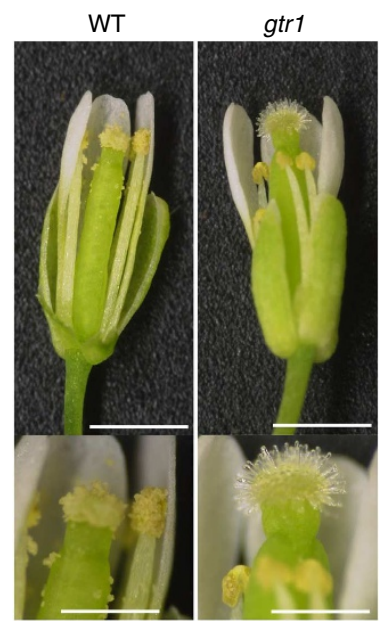

C

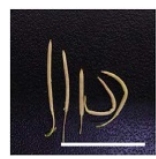

Figure 3 | gtr1 phenotypes in flower. (a) The gtr1 mutant exhibited decreased seed production. The top panel shows representative primary inflorescences. The bottom panel shows representative siliques. Scale bar, $10 \mathrm{~mm}$. (b) Phenotypes of flowers. Scale bar, $1 \mathrm{~mm}$ (top panel) and $0.5 \mathrm{~mm}$ (bottom panel). (c) gtr1 siliques pollinated with WT pollen. Scale bar, $10 \mathrm{~mm}$.

4MTB in GTR1-expressing oocytes (Fig. 5b). These results reveal that GTR1 imports $\mathrm{GA}_{3}$ and JA-Ile in cells in the absence of glucosinolates but that $4 \mathrm{MTB}$ is preferentially transported compared with $\mathrm{GA}_{3}$ and JA-Ile.

GA rescues the $\boldsymbol{g t r} \mathbf{1}$ fertility phenotype. We hypothesized that GTR1 transports active GA(s) and jasmonate(s) during stamen development. However, the true substrate(s) of the transporter at this developmental stage remained uncertain. We attempted to complement the defect in fertility by treating the flower buds with $50 \mu \mathrm{M} \mathrm{GA}_{3}$, JA-Ile or MeJA. Neither treatment with JA-Ile nor MeJA rescued the defect in fertility. On the other hand, $\mathrm{GA}_{3}$ treatment clearly promoted the growth of siliques, resulting in rescue of the decreased fertility (Fig. 6a). We also treated gtr 1 buds with two combinations of hormones (MeJA $+\mathrm{GA}_{3}$ or JAIle $+\mathrm{GA}_{3}$ ) but observed no statistically significant difference in silique length compared with $\mathrm{GA}_{3}$ treatment (Table 1). MeJA+ $\mathrm{GA}_{3}$ or JA-Ile $+\mathrm{GA}_{3}$ treatment increased the seed number of gtr 1 compared with $\mathrm{GA}_{3}$ treatment (Table 2). These results indicate that GA restores filament elongation and anther dehiscence and that neither JA-Ile nor MeJA alone appreciably affect those phenotypes in gtr1 (Fig. 6b).

Specificity for GAs and the relationship between GA and JA. We analysed the specificity of GTR1 for bioactive and inactive GAs. We attempted to complement the defect in fertility by treating flower buds with $50 \mu \mathrm{M} \mathrm{GA}_{1}$ and $\mathrm{GA}_{4}$, which are the bioactive GAs in Arabidopsis, or $\mathrm{GA}_{8}$, an inactive $\mathrm{GA}$ as a negative control. Both $\mathrm{GA}_{1}$ and $\mathrm{GA}_{4}$ rescued the fertility defect (Fig. 6c). We also analysed the specificity of GTR1 transport activity for bioactive and inactive GAs in Xenopus oocytes. GTR1 was capable of transporting $\mathrm{GA}_{3}$ but no significant transport of $\mathrm{GA}_{1}, \mathrm{GA}_{4}, \mathrm{GA}_{9}$ or $\mathrm{GA}_{20}$ was observed (Fig. 5 and Supplementary Fig. 6). We measured the content of $\mathrm{GA}_{1}$ and $\mathrm{GA}_{4}$ in flowers at 
Table 1 | Effects of hormone treatments on silique lengths.

\begin{tabular}{|c|c|c|c|c|c|c|c|c|c|c|}
\hline Treatment & Mock & $\mathbf{G A}_{3}$ & MeJA & JA-Ile & $\mathbf{G A}_{1}$ & $\mathbf{G A}_{4}$ & $\mathbf{G A}_{8}$ & $\mathbf{M e J A}+\mathbf{G A}_{\mathbf{3}}$ & $\mathbf{J A}-I l e+\mathbf{G A}_{3}$ & Untreated \\
\hline WT & $10.50 \pm 0.85 a$ & $9.30 \pm 0.86$ & $9.95 \pm 1.28$ & $10.00 \pm 0.91$ & $9.25 \pm 1.03$ & $9.50 \pm 0.97$ & $9.30 \pm 1.01$ & $9.75 \pm 0.75$ & $9.45 \pm 1.04$ & - \\
\hline gtr1 & $3.25 \pm 0.63 b$ & $9.20 \pm 1.23 a$ & $3.30 \pm 1.32$ & $3.75 \pm 1.55$ & $8.25 \pm 1.4 d$ & $11.35 \pm 1.18 a$ & $3.35 \pm 0.85$ & $10.45 \pm 1.61 a$ & $9.70 \pm 1.18 a$ & - \\
\hline aos & $2.80 \pm 0.26$ & $7.75 \pm 1.98$ & $12.05 \pm 1.66$ & - & - & - & - & - & - & - \\
\hline$W T^{\star}$ gtr1 & - & - & - & - & - & - & - & - & - & $12.50 \pm 1.56 c$ \\
\hline
\end{tabular}

Mature silique lengths $(\mathrm{mm})$ were measured. Buds were treated with or without $50 \mu \mathrm{M}$ GAs or JAs as indicated. Values are the mean $\pm \mathrm{s} . \mathrm{d}$. of 10 biological replicates. Different letters indicate significant differences $(P<0.05)$. The data were analysed followed by Tukey-Kramer multiple comparison test. WT * gtr1 means gtr1 siliques pollinated with WT pollen.

Table 2 | Effects of hormone treatments on seed production.

\begin{tabular}{|c|c|c|c|c|c|c|c|c|c|c|}
\hline Treatment & Mock & $\mathbf{G A}_{3}$ & MeJA & JA-Ile & $\mathbf{G A}_{\mathbf{1}}$ & $\mathbf{G A}_{4}$ & $\mathbf{G A}_{\mathbf{8}}$ & $\mathbf{M e J A}+\mathbf{G A}_{3}$ & JA-Ile $+\mathbf{G A}_{3}$ & Untreated \\
\hline WT & $40.3 \pm 5.9 a$ & $30.5 \pm 7.9$ & $29.0 \pm 9.7$ & $27.2 \pm 8.6$ & $30.8 \pm 7.8$ & $28.9 \pm 8.4$ & $30.3 \pm 5.5$ & $27.9 \pm 4.5$ & $27.3 \pm 6.1$ & - \\
\hline gtr1 & $4.6 \pm 4.6 b$ & $16.1 \pm 7.4 b$ & $0.7 \pm 2.2 b$ & $1.0 \pm 1.7 b$ & $15.5 \pm 8.7 b$ & $38.9 \pm 11.2 \mathrm{a}$ & $0.8 \pm 1.8 b$ & $24.1 \pm 11.4 c$ & $20.5 \pm 6.9 c$ & - \\
\hline aos & $0 \pm 0$ & $0 \pm 0$ & $36.9 \pm 11.7$ & - & - & - & - & - & - & - \\
\hline opr3 & $0 \pm 0$ & $0 \pm 0$ & $20.8 \pm 10.8$ & - & - & - & - & - & - & - \\
\hline$W T{ }^{*}$ gtr1 & - & - & - & - & - & - & - & - & - & $34 \pm 11.2 a$ \\
\hline
\end{tabular}

The numbers of mature seeds were counted. Buds were treated with or without $50 \mu \mathrm{M}$ GAs or JAs as indicated. Values are the mean \pm s.d. of 10 biological replicates. Different letters indicate significant differences $(P<0.05)$. The data were analysed followed by Tukey-Kramer multiple comparison test. WT * gtr1 means gtr1 seeds pollinated with WT pollen.

stages 12,13 and 14. Each of $\mathrm{GA}_{1}$ and $\mathrm{GA}_{4}$ was reduced during flower development in both WT and gtrl, but the absolute content of each in gtrl was significantly lower than that of WT (Supplementary Fig. 8). As a result, we hypothesize that transport of a bioactive GA(s) is impaired in gtr1.

Early induction of GTR1 expression by MeJA (Fig. 1) implies that JA is upstream of GA in stamen development. To clarify the relationship between GA and JA in this developmental stage, we attempted to complement the male sterile phenotype of the JAdeficient mutants aos and opr3 with $\mathrm{GA}_{3}$. The male sterility of aos and opr 3 is rescued by MeJA treatment ${ }^{18}$. aos and opr 3 siliques became swollen following $\mathrm{GA}_{3}$ treatment, but no mature seeds were found in the siliques (Fig. 7). These results indicated that JA signalling is required for stamen development and is independent of the stimulation of GA signalling, even though GA could partially compensate for a lack of JA function during floral development.

\section{Discussion}

Here we revealed that gtr1 is a knockout mutant of GTR1/ NPF2.10 and shows various phenotypes related to JA and GA function that are especially involved in stamen development. Based on the evidence thus far, we speculate that GTR1 positively regulates filament elongation and anther dehiscence by transporting physiologically important compounds that induce stamen development. GTR1 has been primarily identified as a glucosinolate transporter ${ }^{29}$. Nevertheless, our data showed that 4MTB treatment of gtr1 buds could not rescue the reduced fertility phenotype (Supplementary Tables 2 and 3). In addition, treatment with $4 \mathrm{MTB}$ did not potentiate the effect of $\mathrm{GA}_{3}$ and MeJA to restore the fertility of gtr 1 (Tables 1 and 2, Supplementary Tables 2 and 3). These data could indicate that the phenotypes of gtrl stamens may not result from a lack of glucosinolate transport but rather from a lack of hormone transport.

Although we analysed bioactive and inactive forms of JAs, only the bioactive jasmonate JA-Ile was transported into the Xenopus oocytes by GTR1 (Fig. 5a). However, JA-Ile treatment of gtr1 buds did not rescue the reduced fertility phenotype (Fig. 6a).
GTR1 is capable of transporting only $\mathrm{GA}_{3}$ in Xenopus oocytes, an active form of gibberellin (Supplementary Fig. 6b), and the other bioactive gibberellins $\left(\mathrm{GA}_{1}, \mathrm{GA}_{4}\right)$ were able to rescue the gtr1 phenotype in addition to $\mathrm{GA}_{3}$ (Fig. 6). In particular, $\mathrm{GA}_{4}$ fully complemented the infertility phenotype (Tables 1 and 2). The in vivo substrate for GTR1 remains unclear because we found that only $\mathrm{GA}_{3}$ was significantly transported by GTR1 in Xenopus oocytes; $\mathrm{GA}_{4}$ uptake was observed in oocytes even in the absence of GTR1 expression (Supplementary Fig. 6b), which may be a consequence of simple diffusion into oocytes because $\mathrm{GA}_{4}$ is relatively hydrophobic compared with other active GAs. Thus, greater passive diffusion may have mimicked transportermediated $\mathrm{GA}_{4}$ uptake in oocytes. Recent reports have indicated that posttranslational modification alters a nitrate transporter's affinity for substrates ${ }^{37}$. Such modifications might regulate the in vivo substrate selectivity of GTR1. Despite the ability of GTR1 to transport $\mathrm{GA}_{3}$ and JA-lle in oocytes, this does not constitute absolute proof that this is the reason for the gtrl fertility phenotype. Therefore the in vivo substrate for GTR1 during flower development should be clarified in further work.

Synthesis of bioactive GAs occurs throughout floral bud development ${ }^{36}$. After the establishment of floral organs by stage 7, GA3ox, a gene crucial for the synthesis of bioactive GAs, is expressed in the stamen ${ }^{36}$. Very high levels of bioactive GAs have been measured in rice anthers but not in other floral tissues ${ }^{38,39}$, suggesting that anther tissues (specifically the tapetum) act as the source of GAs in flowers. Our results show that $\mathrm{GA}_{1}$ and $\mathrm{GA}_{4}$ contents were reduced from stage 12 to stage 14 in flower development both in WT and gtrl (Supplementary Fig. 8). As GA inactivation pathways are also tightly regulated by developmental signals ${ }^{40}$, the accumulated bioactive GAs in anthers might be inactivated in these stages, resulting in reduction of the GAs in whole flowers. As GTR1 expressed in the stage 14 filaments (Fig. 4), GTR1 could transport bioactive GAs from anthers to the other floral organs such as filaments. We speculate that transportation of the bioactive GAs is impaired in gtrl and the GAs are more quickly inactivated in the gtrl anther, resulting in more strongly reduced bioactive GA contents compared with WT even in whole flowers (Supplementary Fig. 8). 

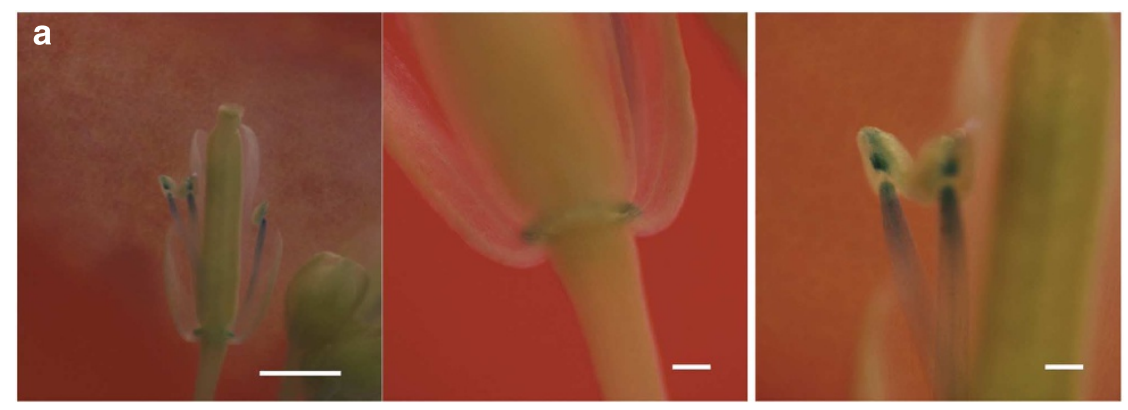

Stage 12

Stage 13

Stage 14
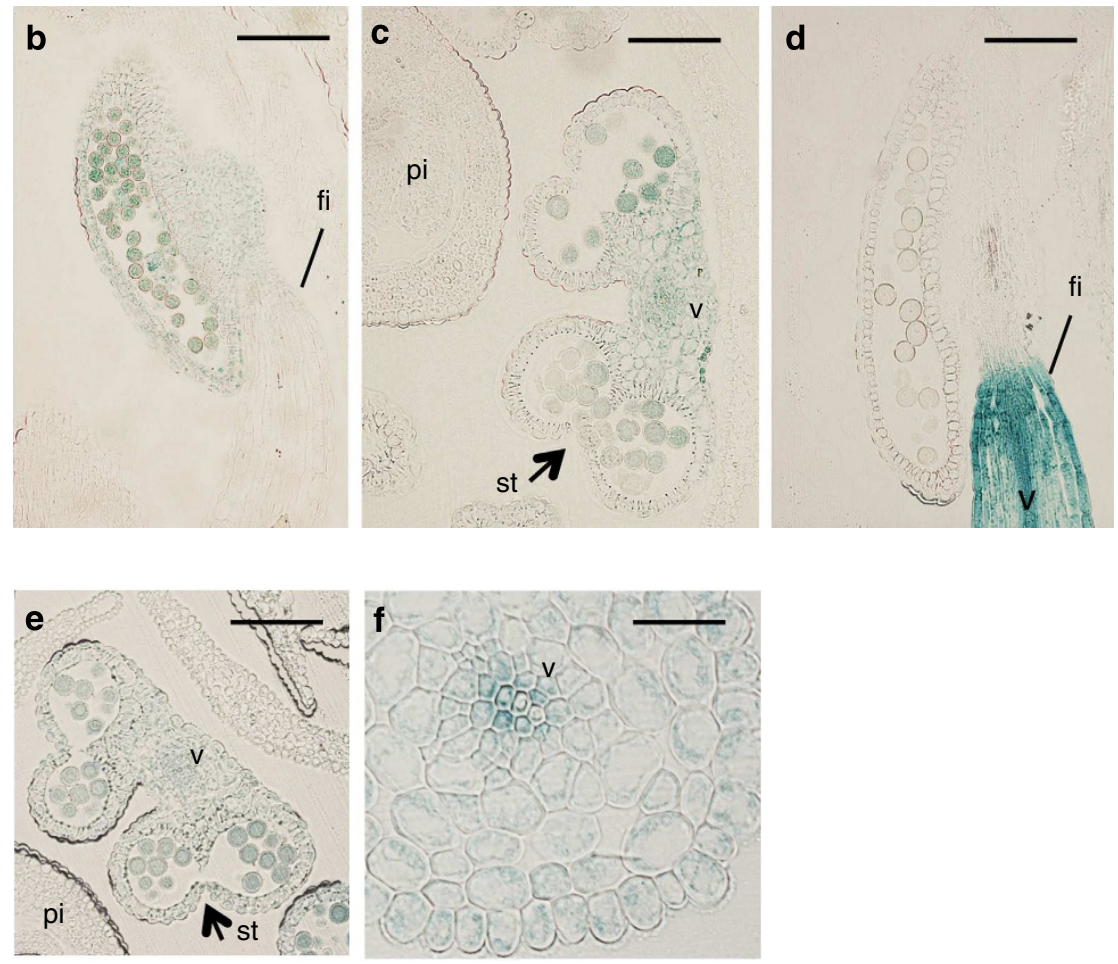

Figure 4 | Localization of GTR1 in floral organs. (a) Histochemical localization of GUS activity in transgenic plants expressing the GUS reporter gene under the control of the GTR1 promoter. GTR1 was expressed in stamen filaments (left panel), base of filaments (middle panel) and anthers (right panel). Scale bar, $1 \mathrm{~mm}$ (left panel) and $100 \mu \mathrm{m}$ (middle and right panels). (b) Vertical section of a proGTR1::GUS flower at stage 12. fi, filament. Scale bar, $100 \mu \mathrm{m}$. (c) Transverse section of a proGTR1::GUS flower at stage 13. pi, pistil; st, stomium; v, vascular bundle. Scale bar, $100 \mu \mathrm{m}$. (d) Vertical section of a proGTR1::GUS flower at stage 14. Scale bar, $100 \mu \mathrm{m}$. (e) Transverse section of a proGTR1::GUS flower at stage 12. Scale bar, $100 \mu \mathrm{m}$. (f) Transverse section of a proGTR1::GUS flower at stage 13. Scale bar, $20 \mu \mathrm{m}$.

We consider $\mathrm{GA}_{4}$ (and $\mathrm{GA}_{1}$ ) may be a substrate of GTR1 in Arabidopsis, and that the decreased supply of these compounds to their principal site of action (perhaps the stamen filaments of stages 13 and 14, as indicated in Fig. 4) may underlie the observed defect in filament elongation. However, detailed localization of bioactive GAs during flower development should be clarified in future.

Because $\mathrm{GA}_{1}$ and $\mathrm{GA}_{4}$ are the major active GAs in Arabidopsis ${ }^{36}$, we suggest the phenotypes observed in gtrl stamens may be caused by specific impairment of the supply of bioactive $\mathrm{GA}_{1}$ and $\mathrm{GA}_{4}$ to such a source tissue. GTR1 transported active GAs in oocytes, but the phenotype of gtrl other than that related to fertility was similar to that of WT, suggesting that other GA transporters may exist. GTR2, a close homologue of GTR1, also transported $\mathrm{GA}_{3}$ into oocytes (Supplementary Fig. 9). This result together with the high GA transport activity of AIT3/ NPF4.1 suggests that several NPF proteins ${ }^{2}$, including NPF2.11 and NPF4.1, may also be involved in GA transport during other developmental processes, and therefore severe developmental abnormalities are not found in the gtrlgtr2 double mutant ${ }^{29}$. On the other hand, specific expression of GTR1 in floral tissues (Fig. 4) is associated with the restricted function of GTR1 during stamen development.

The effects of JA and GA on seed number are probably additive (Table 2). GA 3 treatment of the JA-deficient mutant aos and opr3 induced swelling of siliques, but no mature seeds were produced (Fig. 7a-c). Thus, GA and JA complement each other's function only partially during floral development, and GA signalling is not simply downstream of JA signalling. Rather, these two signalling pathways function in parallel and coordinate with each other during stamen development. GTR1 is expressed in filamentsespecially in vascular bundles-in stages 13 and 14 (Fig. 4d-f), so we suggest that GTR1 may transfer GAs from vascular bundles to cells. Therefore, imported GAs may promote cell expansion to drive filament elongation in stages 13 and 14. On the other hand, GTR1 was expressed less in the tapetum and surrounding tissues 
a

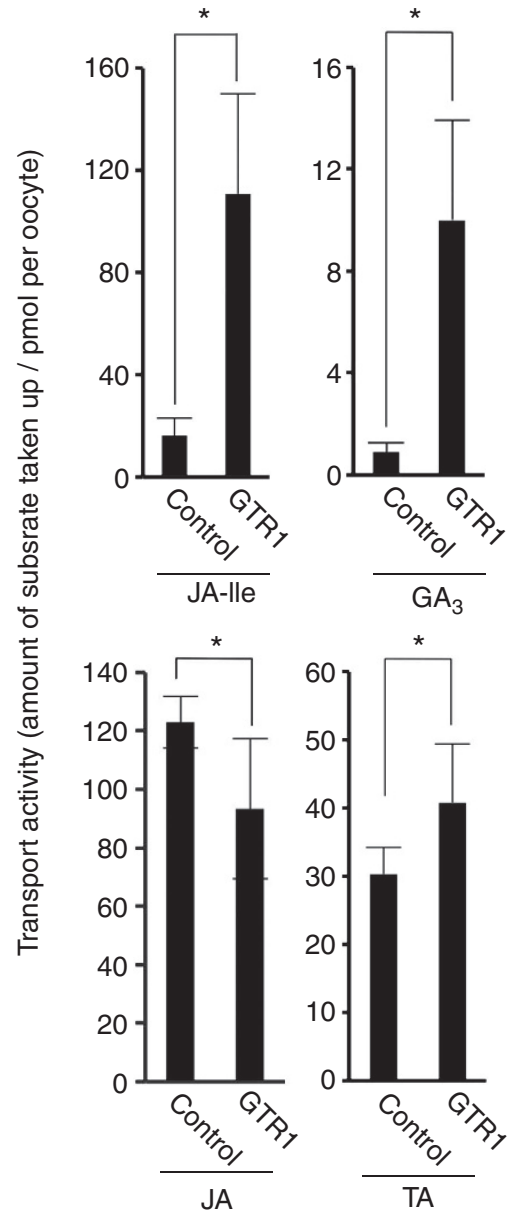

b

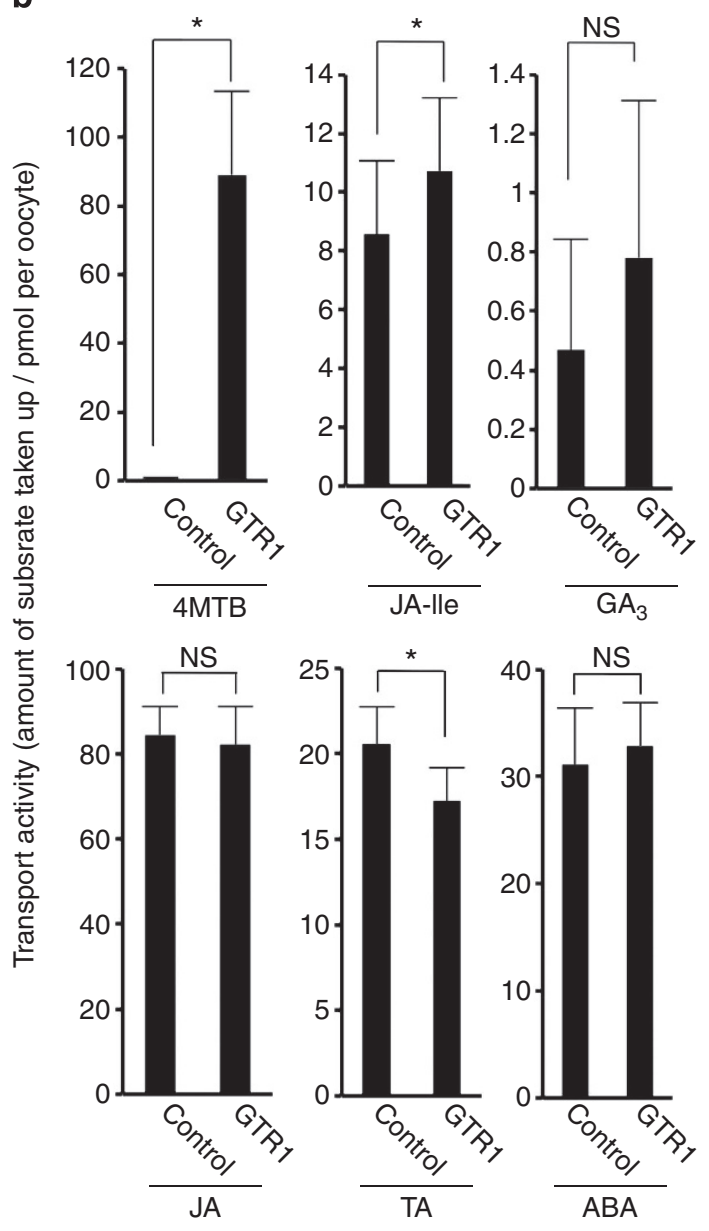

Figure 5 | GTR1-mediated transport of hormones into Xenopus oocytes. (a) GTR1-mediated transport of hormones. Twenty-four hours after cRNA injection, oocytes were added to kulori-based buffer ( $\mathrm{pH}$ 5.0) containing $100 \mu \mathrm{M} \mathrm{JA}, 100 \mu \mathrm{M} \mathrm{TA}, 100 \mu \mathrm{M}$ JA-Ile, $100 \mu \mathrm{M} \mathrm{ABA}$ and $100 \mu \mathrm{M}$ GA 3 and incubated at $17^{\circ} \mathrm{C}$ for $24 \mathrm{~h}$. Oocytes were washed with sorbitol solution, homogenized in extract medium and incubated at $4{ }^{\circ} \mathrm{C}$ for $24 \mathrm{~h}$. After centrifugation, supernatants were collected. Samples were subjected to UPLC/TOFMS analysis. Control means water injection into Xenopus oocytes. Values are the mean \pm s.d. of eight (control) or nine (GTR1) biological replicates. ${ }^{\star} P<0.05$; Student's $t$-test. (b) Activities of GTR1 in the presence of $100 \mu M$ 4MTB. Values are the mean \pm s.d. of 17 biological replicates. ${ }^{\star} P<0.05, N S$, not significant $(P>0.05)$; Student's $t$-test.

that undergo secondary thickening to create the mechanical force necessary for dehiscence. Filament elongation may be a GAregulated process, whereas anther dehiscence may be primarily JA regulated. On the basis of this evidence, we suggest that GTR1 may promote stamen development mainly by supplying GAs to the stamen filaments.

In addition to its possible role in GA transport, GTR1 transports glucosinolates ${ }^{29}$ and JA-Ile in oocytes (our present study), and thus GTR1 may function during various physiological events. GTR1 may be involved in JA signalling (Figs 1 and 2, Supplementary Figs 2 and 10). The gtr1 mutation affected root elongation-an indicator of JA responses-in the presence of MeJA (Supplementary Fig. 10). SAG20 was highly expressed in gtr1 in response to MeJA (Fig. 2). We speculate that in gtr1 leaves, GTR1-dependent hormone transport may be impaired, and this may cause stress-associated senescence, resulting in the degreening phenotype. The reason for SAG12 induction in untreated gtr1 leaves is unknown. Dysregulation of endogenous hormone levels in gtr1 may constitute the molecular basis for senescence.

GA alone induced PDF1.2 expression in WT plants, but this induction was not observed in gtrl (Supplementary Fig. 11). When we compared the effects of the gtrl mutation on PDF1.2 expression following the treatment with GA or MeJA, the expression was higher following GA treatment. We suggest that the compromised PDF1.2 expression observed in gtr1 in the absence of GA supplementation may be mainly caused by the disruption of canonical GA transport in leaves and that impaired GA transport may affect the JA-induced senescence phenotype. In fact, the pif quadruple mutant, which is defective for GA signalling, shows compromised JA responses ${ }^{25}$, leading us to suggest that JA-hypersensitive gtr1 phenotypes may be associated with the absence of GA transport, which could affect the crosstalk between the GA and JA signalling networks ${ }^{23-25}$. However, possible GTR1-mediated JA-Ile transport may be of fundamental importance for these JA-related functions. We found that GTR1 was expressed in shoots, roots and around apical meristems (Supplementary Fig. 12), and a previous report showed that GTR1 is expressed in senescent leaves and that glucosinolates, which are principal substrates for GTR1, are transported from senescent leaves to seeds ${ }^{29}$. Therefore, we speculate that GTR1 may regulate senescence processes in leaves by facilitating hormone transport.

We propose that GTR1/NPF2.10 may be a multifunctional transporter with substrates that are diverse in structure and function. We suggest that GTR1 may have a crucial role in 
a

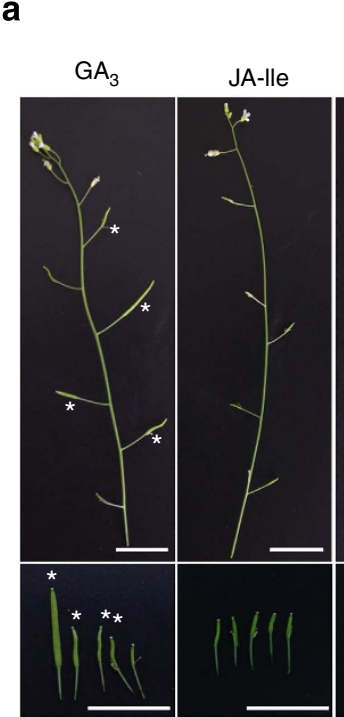

b

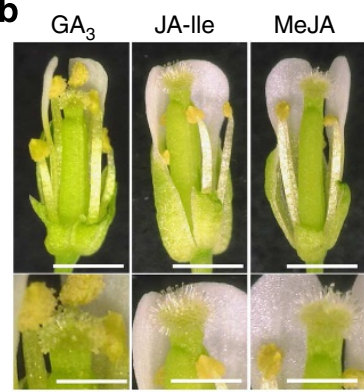

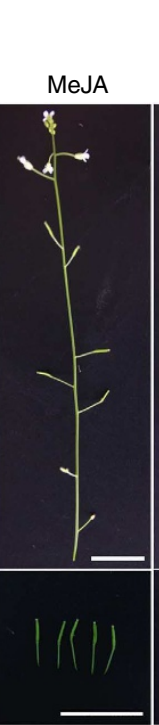

MeJA

$\mathrm{GA}_{3}$

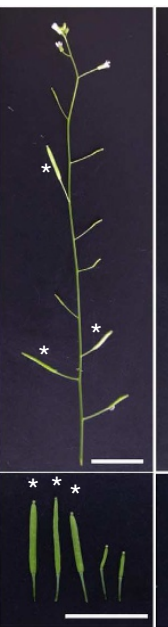

c $\mathrm{GA}_{1}$

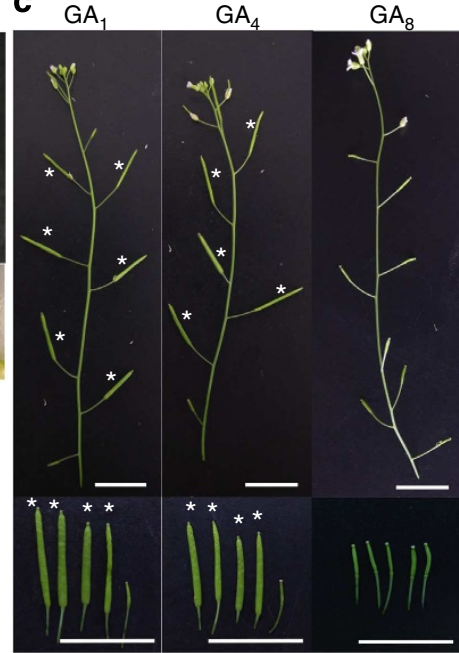

Figure 6 | Effects of GAs on decreased fertility in the gtr1 mutant. (a) gtr1 buds were treated with $50 \mu \mathrm{M} \mathrm{GA}_{3}$, JA-lle or MeJA as indicated. The top panel shows representative primary inflorescences. The bottom panel shows representative siliques. Stars indicate siliques rescued by hormone treatment. Scale bar, $10 \mathrm{~mm}$. (b) Phenotypes of gtr 1 flowers after hormone treatment as indicated. Scale bar, $1 \mathrm{~mm}$ (top panel) and $0.5 \mathrm{~mm}$ (bottom panel). (c) Phenotypes of gtr1 siliques treated with $50 \mu \mathrm{M} \mathrm{GA}, \mathrm{GA}_{4}$ and $\mathrm{GA}_{8}$ as indicated. The top panel shows representative primary inflorescences. The bottom panel shows representative siliques. Stars indicate siliques rescued by hormone treatment. Scale bar, $10 \mathrm{~mm}$.

supplying GA for stamen development and male fertility, a process that is tightly regulated by both JA and GA. We also propose that substrate distribution is an important factor in the activity of this type of multifunctional transporter and raise the possibility that GTR1 also functions in hormone transport in vegetative tissues.

\section{Methods}

Quantitative RT-PCR. Total RNA was isolated from the indicated plant materials using the SV Total RNA Isolation System (Promega KK, Tokyo, Japan). Total RNA (400 ng) was converted to cDNA using PrimeScript RT Enzyme Mix I, Oligo dT Primers and Random 6-mers according to the PrimeScript 1st strand cDNA Synthesis kit protocol (TaKaRa, Shiga, Japan). Quantitative RT-PCR was performed with $8.0 \mathrm{ng}$ cDNA in a final volume of $20 \mu \mathrm{l}$ according to the SYBR Premix Ex Taq II instructions manual (TaKaRa, Shiga, Japan). Quantitative RT-PCR was performed in a Thermal Cycler Dice Real Time System II (TaKaRa, Shiga, Japan).

a

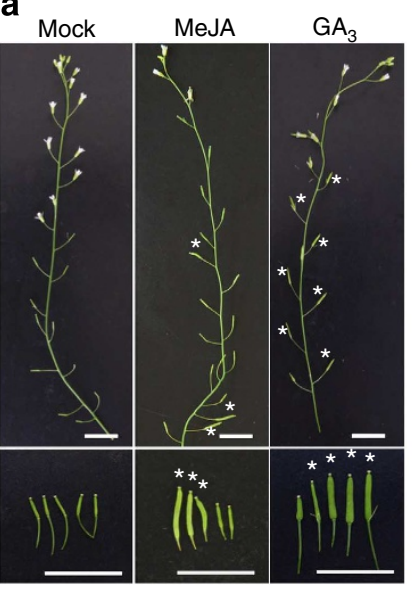

b

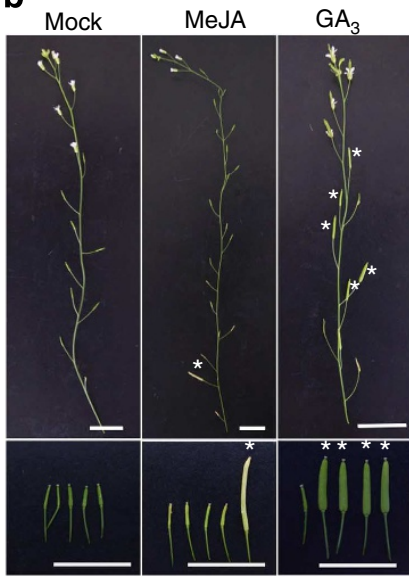

c

MeJA

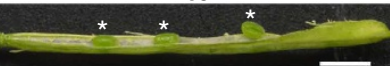

$\mathrm{GA}$

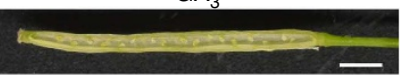

Figure 7 | Effects of GA on JA biosynthesis mutants. (a) aos mutant buds were treated with $0.02 \%$ ethanol (mock), $50 \mu \mathrm{M} \mathrm{MeJA}$ or $50 \mu \mathrm{M} \mathrm{GA}$ as indicated. The top panel shows representative primary inflorescences. The bottom panel shows representative siliques. Stars indicate siliques rescued by hormone treatment. Scale bar, $10 \mathrm{~mm}$. (b) opr3 mutant buds were treated with $0.02 \%$ ethanol (mock), $50 \mu \mathrm{M} \mathrm{MeJA}$ or $50 \mu \mathrm{M} \mathrm{GA}$ as indicated. The top panel shows representative primary inflorescences. The bottom panel shows representative siliques. Stars indicate siliques rescued by hormone treatment. Scale bar, $10 \mathrm{~mm}$. (c) aos siliques rescued by hormone treatment. Stars indicate rescued seeds. Scale bar, $1 \mathrm{~mm}$.

The data were normalized to data for mock-treated WT. The polyubiquitin gene UBQ10 At4g05320 (ref. 41) was used as a reference gene. The thermal cycling conditions were: initial denaturation at $95^{\circ} \mathrm{C}$ for $30 \mathrm{~s}$, followed by 40 cycles of $5 \mathrm{~s}$ at $95^{\circ} \mathrm{C}$ and $30 \mathrm{~s}$ at $60^{\circ} \mathrm{C}$. Three biological replicates were used for each experiment. The primers used were: UBQ10 (At4g05320) forward 5'-GGCCTTGTATAATC CCTGATGAATAAG-3', reverse 5'-AAAGAGATAACAGGAACGGAAACAT AGT-3'; GTR1 (At3g47960) qPCR forward 5'-GTCCATTGGCTGGTATTGCT-3', reverse $5^{\prime}$-ACTTGCTGCAACGTGCATAG-3'; PDF1.2 (AT5G44420) forward $5^{\prime}$-TTTGCTGCTTTCGACGCAC-3' , reverse 5'-CGCAAACCCCTGACCATG-3'; MYC2 (AT1G32640) forward 5'-CGGCTACAACCAACGATGAA-3', reverse $5^{\prime}$-CCGGAGGCCATAAAGTTGAG-3'; ERF1 (AT3G23240) forward 5'-ACGATC CCTAACCGAAAACAGA- $3^{\prime}$, reverse $5^{\prime}$-GTGAGAAGCCGGAGAATGG-3'; ORA59 (AT1G06160) forward 5'-GGCTCTCGCTTATGATCAGG-3', reverse $5^{\prime}$-CCGGAGAGATTCTTCAACGA-3'; LOX3 (AT1G17420) forward 5'-CACTG CAATTCACAAGCAACC- $3^{\prime}$, reverse $5^{\prime}$-CAAAGGAGGAATCGGAGAAGC-3'; AOS (AT5G42650) forward 5'-GCGACGAGAGATCCGAAGA-3', reverse 5' -CTC GCCACCAAAACAACAAA- $3^{\prime}$; DAD1 (AT2G44810) forward $5^{\prime}$-GTGAAGACG AAGAAGAAGAGCAATC- $3^{\prime}$, reverse $5^{\prime}$-GTGAAGACAGCGAAAACGACA TAC-3'; OPR3 (AT2G06050) forward 5' -TTGGACGCAACTGATTCTGAC-3', reverse $5^{\prime}$-GTAGGCGTGGTAGCGAGGTT-3'; SEN4 (AT4G30270) forward $5^{\prime}$-GACTCTTCTCGTGGCGGCGT-3', reverse 5'-CCCACGGCCATTTCCCCA AGC-3'; SAG12 (AT5G45890) forward 5'-GGCGTTTTCAGCGGTTGCGG-3', reverse $5^{\prime}$-CCGCCTTCGCAGCCAAAATCG-3'; SAG18 (AT1G71190) forward $5^{\prime}$-GTTTGCGAGGTGAGAAAATAGGA-3' , reverse $5^{\prime}$-AGAGTAGCATCGTTT GGGTGAAG-3'; SAG20 (AT3G10985) forward 5'-TCGGTAACGTTGTTGCT GGA- $3^{\prime}$, reverse $5^{\prime}$-ACCAAACTCTTTCAAATCGCCA- $3^{\prime}$.

Genotyping and expression analysis of T-DNA knockout plants. T-DNA insertion mutants in the Col-0 background were from The Arabidopsis Biological Resource Center: gtr1, (CS879742). Genotyping analysis was performed with PCR. Primers used were: GTR forward 5'-ATGGAGAGAAAGCCTCTTG-3', reverse 5'-TCAGACAGAGTTCTTGTCT-3'; LB3 5'-TAGCATCTGAATTTCATAACCA ATCTCGA-3'. To produce 35S:GTR1/gtr1 plants, the GTR1 sequence was PCR amplified with PrimeSTAR HS DNA Polymerase (TaKaRa, Shiga, Japan) from cDNA generated using the RNA PCR Kit (TaKaRa, Shiga, Japan) and Gatewaycompatible primers (forward 5'-GGGGACAAGTTTGTACAAAAAAGCAGGCTA CACC- $3^{\prime}$, reverse $5^{\prime}$-GGGGACCACTTTGTACAAGAAAGCTGGGTATCAGAC AGAGTTCTTGTCT-3'). PCR products were cloned into pDONR/Zeo with the Gateway BPII kit (Life Technologies, MD, USA), and sequences were verified. The 
GTR1 constructs were used in Gateway (Life Technologies, MD, USA) LR reactions in combination with the binary expression vectors pGWB2 (ref. 42) and were then checked by sequencing. The resulting construct was then transformed into gtr1 using the Agrobacterium tumefaciens-mediated floral dip method ${ }^{43}$.

Expression analysis was performed with semi-quantitative RT-PCR. For RT-PCR, total RNA was isolated using the SV Total RNA Isolation System (Promega KK, Tokyo, Japan), cDNA was generated using the RNA PCR kit (TaKaRa, Shiga, Japan) and PCR was performed. Actin2 (AT3G18780) was used as the reference gene ${ }^{41}$. Primers used were: GTR1 (AT3G47960) RT-PCR forward 5'-ATGGAGAGAAAGCCTCTTG- ${ }^{\prime}$, reverse 5' -TCAGACAGAGTTCTTG TCT-3'; Actin2 (AT3G18780) forward 5'-ACATTGTGCTCAGTGGTGGA-3', reverse $5^{\prime}$-TCATACTCGGCCTTGGAGAT- $3^{\prime}$.

Chemical reagents. TA was synthesized as previously described. (-)-MeJA was prepared from commercially available racemic MeJA according to the previous method $^{44,45}$. Ozonolysis of olefin moiety in (-)-MeJA, Wittig reaction and successive deprotection gave $\mathrm{TA}^{46}$.

( - )-JA-L-Ile was synthesized according to the previous method ${ }^{47}$. Briefly, racemic MeJA was coupled with L-Ile. After deprotection, the diastereomeric mixture was separated by Silica gel column chromatography to give ( - )-JA-L-Ile.

$( \pm)-J A, M e J A, A B A, G A_{3}$ were purchased from Wako, Osaka, Japan. 4-Methylthiobutylglucosinolate (ChromaDex, CA, USA), $\mathrm{GA}_{1}, \mathrm{GA}_{4}, \mathrm{GA}_{8}, \mathrm{GA}_{9}$ and $\mathrm{GA}_{20}$ (Olchemim, Olomouc, Czech Republic) were also commercially available.

Plant growth conditions. For gene expression analyses, we used 10-day-old seedlings. Ten to fifteen seeds were incubated in a 100-ml Erlenmeyer flask with $30 \mathrm{ml}$ liquid MS medium containing $1 \%$ sucrose under continuous light $\left(20-36 \mu \mathrm{mol} \mathrm{m}-2 \mathrm{~s}^{-1}\right)$ with shaking (130 r.p.m.). Plants were treated with $20 \mu \mathrm{M}$ MeJA or/and $20 \mu \mathrm{M} \mathrm{GA}_{3}$ and harvested at the indicated time points. For CHX treatment, plants were treated with $100 \mu \mathrm{M} \mathrm{CHX}$ for $1 \mathrm{~h}$ before hormone treatment. Plant materials were frozen in liquid nitrogen and stored at $-80^{\circ} \mathrm{C}$ until use. To test sensitivity to MeJA, we used 4-week-old seedlings grown on MS medium containing $50 \mu \mathrm{M} \mathrm{MeJA}, 0.8 \%$ agar and $1 \%$ sucrose under continuous light $\left(30-55 \mu \mathrm{mol} \mathrm{m}{ }^{-2} \mathrm{~s}^{-1}\right)$. We also used the same experimental conditions to measure the root length of plants treated with $20 \mu \mathrm{M}$ MeJA. For the root length measurement, we placed the plates in the vertical orientation. We photographed the roots and measured the root length with ImageJ software (http://rsbweb.nih.gov/ij) ${ }^{48}$. For adult plants, seedlings grown on MS medium containing $0.8 \%$ agar were transferred to the soil and grown to maturity at $22^{\circ} \mathrm{C}$ under continuous light. For hormone complementation assays, buds of adult plants were treated with $50 \mu \mathrm{M}$ GAs, $50 \mu \mathrm{M}$ MeJA or $50 \mu \mathrm{M}$ JA-Ile every 2 days for 2 weeks.

Histochemical analysis. A 2,000-bp, genomic fragment immediately upstream of the GTR1 start codon was amplified with PCR using primers (forward 5'-TGATT ACGCCAAGCTTGGTTCTTAGACTGGCGAG-3' , reverse $5^{\prime}$-ACCTGCAGCCA AGCTTGTCAAGCTTCTCCGCTC- $3^{\prime}$ ) and then cloned into the vector pCambia1391Z. The resulting construct was then transformed into Columbia-0 using the Agrobacterium tumefaciens-mediated floral dip method ${ }^{43}$. GUS staining was performed for $16 \mathrm{~h}$ with $2 \mathrm{mM} \mathrm{X}$-Gluc (5-bromo-4-chloro-3-indolyl- $\beta$-Dglucuronide), $0.5 \mathrm{mM} \mathrm{K}_{4} \mathrm{Fe}(\mathrm{CN})_{6}, 0.5 \mathrm{mM} \mathrm{K}_{3} \mathrm{Fe}(\mathrm{CN})_{6}, 0.3 \%(\mathrm{v} / \mathrm{v})$ Tween 20 and $50 \mathrm{mM} \mathrm{NaPO}_{4}, \mathrm{pH}$ 7.4. The reaction was stopped and plants were destained in $70 \%$ ethanol $^{49}$. To analyse the hormone responses, plants were treated with $20 \mu \mathrm{M}$ MeJA or $0.02 \%$ ethanol for $3 \mathrm{~h}$ before GUS staining. For tissue sectioning, GUS-stained flowers were fixed overnight in FAA solution (5\% v/v formalin, $5 \% \mathrm{v} / \mathrm{v}$ acetic acid, $45 \% \mathrm{v} / \mathrm{v}$ ethanol) at $4{ }^{\circ} \mathrm{C}$.

Fixation of the samples was conducted as described ${ }^{50}$ with minor changes. Samples were dehydrated with an ethanol series, and then ethanol was substituted with mixture containing Technovit 7100 solution (Heraeus Kulzer, Tokyo, Japan) and hardener I (Heraeus Kulzer, Tokyo, Japan). Samples were embedded in a mixture containing hardener II solution (Heraeus Kulzer, Tokyo, Japan), Technovit 7100 solution, and hardener I. The samples were sectioned at $6 \mu \mathrm{m}$ thickness. We photographed the sectioned samples and colour balance of the pictures was adjusted with ImageJ software ${ }^{48}$.

Characterization of the GTR1 transporter using Xenopus oocytes. The GTR1 coding sequence was amplified with primers (forward $5^{\prime}$-TATTAAGCTTGAATT CATGAAGAGCAGAGTCATTCT- $3^{\prime}$, reverse $5^{\prime}$-CGACCCCGGGGAATTCTCA GACAGAGTTCTTGTCT- $3^{\prime}$ ) and subcloned into the EcoRI site of the plasmid, which was previously used to express AtHKT1 (ref. 51). GTR2 was amplified with primers (forward 5'-AAGCTTGAATTCCCCGGGATGGAGAGAAAGCCT CTTG-3', reverse $5^{\prime}$-GGATCCGTCGACCCCGGGTCAGGCAACGTTCTTG TCT $-3^{\prime}$ ) and subcloned into the SmaI site. NPF4.1 was amplified with primers (forward 5'-AAGCTTGAATTCCCCGGGATGCAGATTGAGATGGAAG-3', reverse $5^{\prime}$-GGATCCGTCGACCCCGGGCTAATATCTTTTCGCCCAG-3') and subcloned into the SmaI site. Capped complementary RNA (cRNA) of each gene was prepared using the mMESSAGE mMACHINE kit (Life Technologies, MD, USA). Oocyte preparation and cRNA injection were performed as described ${ }^{51}$. Each oocyte was injected with $500 \mathrm{ng}$ cRNA. After incubation for 24 or $48 \mathrm{~h}$, the buffer was replaced with $100 \mu \mathrm{l}$ of kulori-based solution ${ }^{51}(90 \mathrm{mM}$ sodium gluconate, $1 \mathrm{mM}$ potassium gluconate, $1 \mathrm{mM}$ calcium gluconate, $1 \mathrm{mM}$ magnesium gluconate, $1 \mathrm{mM} \mathrm{LaCl}_{3}$ and $10 \mathrm{mM}$ MES, pH 5) containing the corresponding substrate(s), and oocytes were incubated for an additional $24 \mathrm{~h}$. For Determination of $K_{m}$ values, $24 \mathrm{~h}$ after cRNA injection, two oocytes were treated with the $\mathrm{GA}_{3}$ substrate $(1,10,30,60,100,300,1,000$ and 2,000 $\mu \mathrm{M})$ in a Kulori-based buffer (pH 5) and incubated at $17^{\circ} \mathrm{C}$ for $24 \mathrm{~h}$.

Sample preparation for liquid chromatography/mass spectrometry. Oocytes were washed twice with $200 \mathrm{mM}$ sorbitol solution, homogenized in $40 \mu \mathrm{l}$ extract buffer (28\% methanol, $0.05 \%$ acetic acid) and incubated at $4{ }^{\circ} \mathrm{C}$ for $24 \mathrm{~h}$. After centrifugation $(20,000 \mathrm{~g}$, room temperature, $20 \mathrm{~min})$, supernatants were collected, and 10- $\mu \mathrm{l}$ samples were subjected to ultra-performance liquid chromatography coupled to time-of-flight mass spectrometry (UPLC/TOFMS) analysis.

UPLC/TOFMS analysis. UPLC/TOFMS analysis of $10-\mu$ samples from oocyte extracts was performed using an Agilent 1290 Infinity (Agilent Technologies, CA, USA) coupled to a microTOF II (Bruker Daltonics, Leipzig, Germany). A ZORBAX Eclipse Plus C18 column $(1.8 \mu \mathrm{m}, 2.1 \times 50 \mathrm{~mm}$; Agilent Technologies) was used to separate substrates. The mobile phases were: A, $20 \%(\mathrm{v} / \mathrm{v})$ aqueous methanol with $0.05 \%(\mathrm{v} / \mathrm{v})$ acetic acid, and B, methanol with $0.05 \%(\mathrm{v} / \mathrm{v})$ acetic acid. The gradient programme was: 0 to $3.5 \mathrm{~min}$, isocratic $90 \% \mathrm{~A} ; 3.5$ to $6 \mathrm{~min}$, linear gradient 90 to $0 \% \mathrm{~A} ; 6.1 \mathrm{~min}$ to $9 \mathrm{~min}$, isocratic $90 \% \mathrm{~A}$, with a flow rate of $0.15 \mathrm{ml} \mathrm{min}^{-1}$. The mass spectrometer was run in the negative mode with scan range $100-700 \mathrm{~m} / \mathrm{z}$. The capillary voltage was $4,200 \mathrm{~V}$, the nebulizer gas pressure was 1.6 bar, the desolvation gas flow was $8.01 \mathrm{~min}^{-1}$, and the temperature was $180^{\circ} \mathrm{C}$. The substrates were quantified on the basis of an extracted ion chromatogram and the corresponding peak position of the standard solution.

EGFP fusion and subcellular localization. The stop codon of GTR1 was mutagenized with PCR to introduce a SalI site with primers (forward $5^{\prime}$-TACAATTAC AGTCGACATGAAGAGCAGAGTCATTCT- ${ }^{\prime}$, reverse $5^{\prime}$-ATCCTCTAGAGTC GACGACAGAGTTCTTGTCT- $3^{\prime}$ ). This site was then used to make an in-frame GTR1-EGFP fusion construct. The final construct, 35S:GTR1-EGFP-pUC18, and the empty vector, 35S:EGFP-pUC18, were transiently expressed in onion epidermal cells using a particle gun-mediated system (PDS-1000/He; Bio-Rad, CA, USA). The bombarded cells were kept in the dark at $22^{\circ} \mathrm{C}$ for $12 \mathrm{~h}$ followed by GFP imaging using confocal microscopy (Leica; DM2500).

Pollen germination in vitro. The in vitro pollen germination experiment was conducted as described ${ }^{52}$ with minor modifications. For WT and gtr1, stage 14 flowers were randomly harvested from eight plants each. For each experiment, pollen grains were then obtained from three separate flowers. The medium for pollen germination contained $5 \mathrm{mM}$ MES-Tris ( $\mathrm{pH} 5.8$ ), $1 \mathrm{mM} \mathrm{KCl}, 0.8 \mathrm{mM}$ $\mathrm{MgSO}_{4}, 1.5 \mathrm{mM}$ boric acid, $10 \mathrm{mM} \mathrm{CaCl}_{2}, 16 \%$ (w/v) sucrose and $0.5 \%$ (w/v) INA agar. Pollen germination was observed by microscopy after $24 \mathrm{~h}$ incubation at $23^{\circ} \mathrm{C}$ in continuous light $\left(30 \mu \mathrm{mol} \mathrm{m}{ }^{-2} \mathrm{~s}^{-1}\right)$ with high humidity, and the number of germinated pollen grains was counted.

Plant hormone quantification. The developmental stages of flowers were as described ${ }^{35}$. Flowers were categorized into three stages (12-14) and harvested every 3 days from 4- to 6-week-old plants grown on soil. Flower samples ( $\sim 500 \mathrm{mg}$ fresh weight per stage) were frozen in liquid nitrogen and stored at $-80^{\circ} \mathrm{C}$. Frozen samples from WT and gtrl were lyophilized, and GAs were extracted from the samples using $80 \%(\mathrm{v} / \mathrm{v})$ acetone containing $1 \%(\mathrm{v} / \mathrm{v})$ acetic acid as described ${ }^{53}$ Briefly, lyophilized plant materials (12-28 mg dry weight) were ground in $80 \%$ $(\mathrm{v} / \mathrm{v})$ acetone containing $1 \%(\mathrm{v} / \mathrm{v})$ acetic acid with $\left[17,1-^{2}{ }^{2} \mathrm{H}_{2}\right] \mathrm{GAs}(300 \mathrm{pg}$ each) as internal standards. Crude extract was purified through polyvinylpyrrolidone (500 mg; Tokyo Kasei) and reverse-phase (Oasis HLB, $60 \mathrm{mg}$; Waters) cartridges. The GA-containing fraction was further purified by an ion-exchange column (Bond Elut DEA, $100 \mathrm{mg}$; Agilent) and a SepPak silica cartridge (100 mg; Waters). Obtained GA-enriched fraction was concentrated to dryness, dissolved in $20 \mu \mathrm{l}$ of water containing $1 \%$ acetic acid, and then subjected to LC-tandem mass spectrometry (LC-MS/MS) analysis.

GAs were quantified as described ${ }^{53}$ with some modifications. LC was performed with an Acquity UPLC HSS T3 column $(1.8 \mu \mathrm{m}, 2.1 \times 50 \mathrm{~mm}$; Waters, MA, USA $)$ using the following programme with solvent $\mathrm{A}$ (water containing $0.01 \%(\mathrm{v} / \mathrm{v})$ acetic acid) and solvent $B$ (acetonitrile containing $0.05 \%(v / v)$ acetic acid): isocratic flow with $3 \% \mathrm{~B}$ for $30 \mathrm{~s}$, a linear gradient of B from 3 to $12 \%$ over $2.5 \mathrm{~min}$, a linear gradient of B from 12 to $25 \%$ over 5 min, a linear gradient of B from 25 to $40 \%$ over 4 min, isocratic flow with $40 \%$ of B for 2 min, a linear gradient of B from 40 to $98 \%$ over $1 \mathrm{~min}$, and isocratic elution with $98 \% \mathrm{~B}$ for $1 \mathrm{~min}$. MS/MS conditions were as follows: ion spray voltage floating $(\mathrm{kV})=-4.0$, desolvation temperature $=600^{\circ} \mathrm{C}$, collision energy $(\mathrm{V})=-40\left(\mathrm{GA}_{1}\right)$ or $-30\left(\mathrm{GA}_{4}\right)$, declustering potential $=-90$, MS/MS transition $(\mathrm{m} / \mathrm{z}): 349.2 / 275.2\left(\left[{ }^{2} \mathrm{H}_{2}\right] \mathrm{GA1}\right), 347.2 / 273.2\left(\mathrm{GA}_{1}\right), 333.2 / 259.2$ $\left(\left[{ }^{2} \mathrm{H}_{2}\right] \mathrm{GA}_{4}\right), 331.2 / 257.2\left(\mathrm{GA}_{4}\right)$. The LC retention time was $5.5 \mathrm{~min}$ for $\mathrm{GA}_{1}$ and $11.5 \mathrm{~min}$ for $\mathrm{GA}_{4}$. 


\section{References}

1. Petrásek, J. \& Friml, J. Auxin transport routes in plant development. Development 136, 2675-2688 (2009).

2. Léran, S. et al. A unified nomenclature of NITRATE TRANSPORTER 1/PEPTIDE TRANSPORTER family members in plants. Trends Plant Sci. 19, 5-9 (2013).

3. Krouk, G. et al. Nitrate-regulated auxin transport by NRT1. 1 defines a mechanism for nutrient sensing in plants. Dev. Cell 18, 927-937 (2010)

4. Kang, J. et al. PDR-type ABC transporter mediates cellular uptake of the phytohormone abscisic acid. Proc. Natl Acad. Sci. USA 107, 2355-2360 (2010).

5. Kuromori, T. et al. ABC transporter AtABCG25 is involved in abscisic acid transport and responses. Proc. Natl Acad. Sci. USA 107, 2361-2366 (2010).

6. Kanno, Y. et al. Identification of an abscisic acid transporter by functional screening using the receptor complex as a sensor. Proc. Natl Acad. Sci. USA 109, 9653-9658 (2012).

7. Zhang, H. et al. A DTX/MATE-type transporter facilitates abscisic acid efflux and modulates ABA sensitivity and drought tolerance in Arabidopsis. Mol. Plant 7, 1522-1532 (2014)

8. Ko, D. et al. Arabidopsis ABCG14 is essential for the root-to-shoot translocation of cytokinin. Proc. Natl Acad. Sci. USA 111, 7150-7155 (2014)

9. Zhang, K. et al. Arabidopsis ABCG14 protein controls the acropetal translocation of root-synthesized cytokinins. Nat. Commun. 5, 3274 (2014).

10. Kretzschmar, T. et al. A petunia ABC protein controls strigolactone-dependent symbiotic signalling and branching. Nature 483, 341-344 (2012).

11. Peng, J. Gibberellin and jasmonate crosstalk during stamen development. J. Int. Plant Biol. 51, 1064-1070 (2009).

12. Sun, T. P. Gibberellin-GID1-DELLA: a pivotal regulatory module for plant growth and development. Plant Physiol. 154, 567-570 (2010).

13. Swain, S. M. \& Singh, D. P. Tall tales from sly dwarves: novel functions of gibberellins in plant development. Trends Plant Sci. 10, 123-129 (2005)

14. Acosta, I. F. \& Farmer, E. E. in The Arabidopsis book (eds Somerville, C. R. \& Meyerowitz, E. M.) (American Society of Plant Biologists, 2010).

15. Wasternack, C., Forner, S., Strnad, M. \& Hause, B. Jasmonates in flower and seed development. Biochimie. 95, 79-85 (2013).

16. Schilmiller, A. L. \& Howe, G. A. Systemic signaling in the wound response. Curr. Opin. Plant Biol. 8, 369-377 (2005).

17. Browse, J. Jasmonate passes muster: a receptor and targets for the defense hormone. Annu. Rev. Plant Biol. 60, 183-205 (2009).

18. Thines, B., Mandaokar, A. \& Browse, J. Characterizing jasmonate regulation of male fertility in Arabidopsis. Methods Mol. Biol. 1011, 13-23 (2013).

19. Koornneef, M. \& Van Der Veen, J. H. Induction and analysis of gibberellin sensitive mutants in Arabidopsis thaliana (L.) Heynh. Theor. Appl. Genet. 58, 257-263 (1980)

20. Sun, T. P., Goodman, H. M. \& Ausubel, F. M. Cloning the Arabidopsis GA1 locus by genomic subtraction. Plant Cell 4, 119-128 (1992).

21. Sun, T. P. in The Arabidopsis Book (eds Somerville, C. R. \& Meyerowitz, E. M.) (American Society of Plant Biologists, 2008).

22. Cheng, H. et al. Gibberellin regulates Arabidopsis floral development via suppression of DELLA protein function. Development 131, 1055-1064 (2004).

23. Hou, X., Lee, L. Y., Xia, K., Yan, Y. \& Yu, H. DELLAs modulate jasmonate signaling via competitive binding to JAZs. Dev. Cell 19, 884-894 (2010).

24. Hong, G. J., Xue, X. Y., Mao, Y. B., Wang, L. J. \& Chen, X. Y. Arabidopsis MYC2 interacts with DELLA proteins in regulating sesquiterpene synthase gene expression. Plant Cell 24, 2635-2648 (2012).

25. Yang, D. et al. Plant hormone jasmonate prioritizes defense over growth by interfering with gibberellin signaling cascade. Proc. Natl Acad. Sci. USA 109, E1192-E1200 (2012).

26. Sasaki-Sekimoto, Y. et al. Basic helix-loop-helix transcription factors JASMONATE-ASSOCIATED MYC2-LIKE1 (JAM1), JAM2, and JAM3 are negative regulators of jasmonate responses in Arabidopsis. Plant Physiol. 163, 291-304 (2013)

27. Obayashi, T., Nishida, K., Kasahara, K. \& Kinoshita, K. ATTED-II updates: condition-specific gene coexpression to extend coexpression analyses and applications to a broad range of flowering plants. Plant Cell Physiol. 52, 213-219 (2011)

28. Obayashi, T. \& Kinoshita, K. Rank of correlation coefficient as a comparable measure for biological significance of gene coexpression. DNA Res. 16, 249-260 (2009).

29. Nour-Eldin, H. H. et al. NRT/PTR transporters are essential for translocation of glucosinolate defence compounds to seeds. Nature 488, 531-534 (2012)

30. Lorenzo, O., Chico, J. M., Sánchez-Serrano, J. J. \& Solano, R. JASMONATEINSENSITIVE1 encodes a MYC transcription factor essential to discriminate between different jasmonate-regulated defense responses in Arabidopsis. Plant Cell 16, 1938-1950 (2004).

31. Thines, B. et al. JAZ repressor proteins are targets of the SCFCOI1 complex during jasmonate signalling. Nature 448, 661-665 (2007).

32. Chini, A. et al. The JAZ family of repressors is the missing link in jasmonate signalling. Nature 448, 666-671 (2007).
33. Yan, Y. et al. A downstream mediator in the growth repression limb of the jasmonate pathway. Plant Cell 19, 2470-2483 (2007).

34. Weaver, L. M., Gan, S., Quirino, B. \& Amasino, R. M. A comparison of the expression patterns of several senescence-associated genes in response to stress and hormone treatment. Plant Mol. Biol. 37, 455-469 (1998).

35. Smyth, D. R., Bowman, J. L. \& Meyerowitz, E. M. Early flower development in Arabidopsis. Plant Cell 2, 755-767 (1990).

36. Plackett, A. R., Thomas, S. G., Wilson, Z. A. \& Hedden, P. Gibberellin control of stamen development: a fertile field. Trends Plant Sci. 16, 568-578 (2011).

37. Liu, K. H. \& Tsay, Y. F. Switching between the two action modes of the dualaffinity nitrate transporter CHL1 by phosphorylation. EMBO J. 22, 1005-1013 (2003).

38. Hirano, K. et al. Comprehensive transcriptome analysis of phytohormone biosynthesis and signaling genes in microspore/pollen and tapetum of rice. Plant Cell Physiol. 49, 1429-1450 (2008).

39. Kanno, Y. et al. Comprehensive hormone profiling in developing Arabidopsis seeds: examination of the site of ABA biosynthesis, ABA transport and hormone interactions. Plant Cell Physiol. 51, 1988-2001 (2010).

40. Yamaguchi, S. Gibberellin metabolism and its regulation. Annu. Rev. Plant Biol. 59, 225-251 (2008)

41. Czechowski, T., Stitt, M., Altmann, T., Udvardi, M. K. \& Scheible, W. R. Genome-wide identification and testing of superior reference genes for transcript normalization in Arabidopsis. Plant Physiol. 139, 5-17 (2005).

42. Nakagawa, T. et al. Development of series of gateway binary vectors, pGWBs, for realizing efficient construction of fusion genes for plant transformation. J. Biosci. Bioeng. 104, 31-41 (2007).

43. Berberich, T., Takahashi, Y., Saitoh, H. \& Terauchi, R. in The Handbook of Plant Functional Genomics (eds Kahl, G. \& Meksem, K.) Ch. 6, 113-136 (Wiley, 2008).

44. Kiyota, H., Higashi, E., Koike, T. \& Oritani, T. Lipase-catalyzed preparation of both enantiomers of methyl jasmonate. Tetrahedron Asymmetry 12, 1035-1038 (2001).

45. Asamitsu, Y., Nakamura, Y., Ueda, M., Kuwahara, S. \& Kiyota, H. Synthesis and odor description of both enantiomers of methyl 4,5-didehydro-jasmonate a component of jasmin absolute. Chem. Biodivers. 3, 654-659 (2006).

46. Nakamura, Y., Miyatake, R., Inomata, S. \& Ueda, M. Synthesis and bioactivity of potassium $\beta$-D-glucopyranosyl 12-hydroxy jasmonate and related compounds. Biosci. Biotechnol. Biochem. 72, 2867-2876 (2008).

47. Kramell, R., Schmidt, J., Schneider, G., Sembdner, G. \& Schreiber, K. Synthesis of $N$-(jasmonoyl)amino acid conjugates. Tetrahedron 44, 5791-5807 (1988)

48. Abramoff, M. D., Magelhaes, P. J. \& Ram, S. J. Image processing with ImageJ. Biophotonics Int. 11, 36-42 (2004).

49. Jefferson, R. A., Kavanagh, T. A. \& Bevan, M. W. GUS fusions: betaglucuronidase as a sensitive and versatile gene fusion marker in higher plants EMBO J. 20, 3901-3907 (1987).

50. Adachi, S., Nobusawa, T. \& Umeda, M. Quantitative and cell type-specific transcriptional regulation of A-type cyclin-dependent kinase in Arabidopsis thaliana. Dev. Biol. 329, 306-314 (2009).

51. Uozumi, N. et al. The Arabidopsis HKT1 gene homolog mediates inward $\mathrm{Na}^{+}$ currents in xenopus laevis oocytes and $\mathrm{Na}^{+}$uptake in Saccharomyces cerevisiae. Plant Physiol. 122, 1249-1259 (2000).

52. Fan, L. M., Wang, Y. F., Wang, H. \& Wu, W. H. In vitro Arabidopsis pollen germination and characterization of the inward potassium currents in Arabidopsis pollen grain protoplasts. J. Exp. Bot. 52, 1603-1614 (2001)

53. Yamaguchi, N. et al. Gibberellin acts positively then negatively to control onset of flower formation in Arabidopsis. Science 344, 638-641 (2014).

\section{Acknowledgements}

We thank E. E. Farmer for critical reading of our manuscript. We also thank M. Shimojima, K. Hori and other Ohta \& Masuda lab members for critical comments and/or technical help. Full-length cDNAs were provided by RIKEN Bio Resource Center (BRC) This work was supported in part by the Global COE Program (From the Earth to 'Earths') for H.S. and H.O., a Grant-in-Aid for Scientific Research (No.24580135 and 26102711 to S.H, 25119725 to M.S. and 25292055 to N.U.), the Botanical Research Grant by the New Technology Development Foundation for Y.I. and a Grant-in-Aid for Scientific Research (No.23102012) on Innovative Areas Chemical Biology of Natural Products" (No.2301) for M.U. from MEXT, Japan. Hormonome analysis was supported by the Japan Advanced Plant Science Network.

\section{Author contributions}

H.S. performed gene expression analyses and subcellular localization experiments. H.S. and M.K.-S. performed physiological analyses and histochemical localization experiments. H.S., T.U., J.C. and M.K.-S. produced transgenic lines. T.O., S.H., and Y.I. of Tohoku University and M.S. of RIKEN characterized the GTR1 transport activity. H.S. and M.K.-S. of Tokyo Institute of Technology and Y. Kanno and M.S. of RIKEN measured the GA content. H.S., Y.S.-S., S.M. and H.O. of Tokyo Institute of Technology, N.U. and M.U. of Tohoku University and M.S. and Y. Kamiya of RIKEN designed most of the experiments and discussed the results. H.S., S.H., Y.I., Y.S.-S., M.K.-S. and H.O. 
wrote the manuscript. H.O. planned the project. All the authors discussed the results and commented on the manuscript.

\section{Additional information}

Supplementary Information accompanies this paper at http://www.nature.com/

naturecommunications

Competing financial interests: The authors declare no competing financial interests.

Reprints and permission information is available online at http://npg.nature.com/ reprintsandpermissions/
How to cite this article: Saito, H. et al. The jasmonate-responsive GTR1 transporter is required for gibberellin-mediated stamen development in Arabidopsis. Nat. Commun. 6:6095 doi: 10.1038/ncomms7095 (2015).

(c) (i) This work is licensed under a Creative Commons Attribution 4.0 International License. The images or other third party material in this article are included in the article's Creative Commons license, unless indicated otherwise in the credit line; if the material is not included under the Creative Commons license, users will need to obtain permission from the license holder to reproduce the material. To view a copy of this license, visit http://creativecommons.org/licenses/by/4.0/ 